\title{
A REPRESENTATION THEOREM FOR FUNCTION ALGEBRAS WITH APPLICATION TO ALMOST PERIODIC FUNCTIONS
}

\author{
K. E. AUBERT
}

1. Introduction. The intimate connection between the almost periodic functions ${ }^{1}$ on an arbitrary group $G$ and the continuous functions on a corresponding compact group $\bar{G}$ was first established by Weil in [4]. He showed that to any topological group $G$ one may canonically associate a compact group $\bar{G}$ containing as a dense subgroup a continuous and homomorphic image $\varphi(G)$ of $G$ such that the function $f$ on $G$ is almost periodic if and only if there exists a continuous function $g$ on $\bar{G}$ such that

$$
f(x)=g(p(x)) \quad \text { for all } \quad x \in G .
$$

Based on the Gelfand-Neumark theorem for commutative $B^{*}$-algebras Loomis in [3] was able to derive this connection within the scope of general Banach algebra theory. The purpose of the present note is to point out that Loomis' treatment of almost periodic functions can also be achieved by an alternative use of the maximal ideal method using maximal convex lattice-closed subspaces in the real ordered vector space $A^{R}(G)$ of all real-valued almost periodic functions on $G$ instead of the usual maximal ideals in the Banach algebra $A^{C}(G)$ of all complex-valued almost periodic functions on $G$. By this procedure-which is well known from spectral theory - the Loomis approach to almost periodic functions is freed from the use of the Gelfand-Neumark theorem and any other Banach algebra theory, and is made to depend on simpler representation theorems of Kadison [2]. In fact what we shall do is to give two direct proofs of the Gelfand-Neumark theorem for a certain wide class of function algebras which includes the algebra $A^{C}(G)$ as a special case. The first proof is based on one of the simpler representation theorems of Kadison. The proof given by Kadison in [2] of the representation theorem in question depends (apart from the unavoidable Stone-Weier-

Received March 1, 1959.

1 In the non-commutative case "almost periodic" should for instance be interpreted as "left almost periodic". 
strass theorem) on the Krein-Milman theorem, but is nevertheless of a simpler nature than the usual proof of the Gelfand-Neumark theorem. Our second proof is perhaps of more interest since it also avoids the use of the Krein-Milman theorem and only depends on an exceedingly simple representation theorem for ordered vector spaces together with elementary topological arguments.

\section{The Gelfand-Neumark theorem for $F^{*}$-algebras. The first proof.} Let $A$ denote a complex Banach algebra of complex-valued, bounded and continuous functions on a topological space $X$. The operations are pointwise and the norm is the uniform norm. We shall say that $A$ is an $F^{*}$-algebra if $A$ contains the constant functions and is closed under the operations of taking conjugates $f \rightarrow \bar{f}$ and absolute values $f \rightarrow|f|$. The family $A^{R}$ of all real-valued functions in $A$ clearly forms a real latticeordered vector space (or algebra). The family of maximal ideals of $A$ will be denoted by $\mathfrak{M}$ and the family of maximal convex subspaces of $A^{R}$ which are lattice-closed by $\mathfrak{R}$. Considering $A^{R}$ as an ordered algebra we shall denote by $\mathfrak{P}$ the subset of $\mathfrak{A}$ consisting of those subspaces which are at the same time ideals. For all the definitions and notations (like the $\hat{f}$-notation) which have their background in general Banach algebra theory we must refer the reader to [3]. With respect to ordered vector spaces and algebras one can use [2] as a standard reference. We should, however, like to remind the reader of a convention which is essential in the following. Whereas there is a one-to-one correspondence between maximal ideals and multiplicative functionals in Banach algebra theory there is no one-to-one correspondence between maximal convex subspaces and positive functionals in the ordered theory. One-to-one correspondence is, however, restored if we adjust the positive functionals in such a way that they all take the value 1 on the order unit.

Before entering the proper subject matter of the paper it is perhaps worth while remarking that the Gelfand-Neumark theorem immediately implies that $\mathfrak{M}$ and $\mathfrak{R}$ are homeomorphic if they are both equipped with the Gelfand topology. Since this is equally simple to see for general $B^{*}$-algebras we suppose for the moment that $A$ is a commutative $B^{*}$ algebra with an identity. By the Gelfand-Neumark theorem

$$
A \cong C(\mathfrak{M})
$$

and this isomorphism has a restriction to the real ordered vector space $A^{R}$ of self-adjoint elements of $A$ and the ordered vector space $C^{R}(\mathfrak{M})$ which is an order isomorphism

$$
A^{R} \cong C^{R}(\mathfrak{M}) \text {. }
$$


In $C^{R}(\mathfrak{M})$ there are no other maximal convex lattice-closed subspaces than those corresponding to points in $\mathfrak{M}$, so that if $\mathfrak{N}^{\prime}$ denotes the family of maximal convex lattice-closed subspaces of $C^{R}(\mathfrak{M})$ we shall have

$$
C^{R}(\mathfrak{M}) \cong C^{R}\left(\mathfrak{R}^{\prime}\right) \text {. }
$$

By the isomorphism $A^{R} \cong C^{R}(\mathfrak{M})$ there is a one-to-one correspondence between $\mathfrak{N}$ and $\mathfrak{N}^{\prime}$ such that if the Gelfand topology of $\mathfrak{R}^{\prime}$ is transferred by this correspondence to $\mathfrak{N}$ it gives the Gelfand topology in $\mathfrak{N}$ and

$$
C^{R}(\mathfrak{M}) \cong C^{R}(\mathfrak{R}) \text {. }
$$

It is well-known by using the Stone topology that this order isomorphism induces a homeomorphism between $\mathfrak{M}$ and $\mathfrak{R}$.

The above remark shows already that we may equally well use $\mathfrak{R}$ instead of $\mathfrak{M}$ as the compact group $\bar{G}$ in Weil's theorem.

We shall now give the first proof of the following

Theorem. Any $F^{*}$-algebra $A$ is isomorphic and isometric with the algebra $C(\mathfrak{M})$ of all complex-valued continuous functions on its maximal ideal space $\mathfrak{M}$ equipped with the Gelfand topology.

REMarK. The reader will perhaps object that the Gelfand-Mazur theorem is already implicit in the formulation of this theorem by the very definition of the Gelfand topology,-and therefore contradicts our promise to give proofs which are free from Banach algebra theory. It will, however, be clear from the proof that this is not the case. In fact the present proof also contains a direct proof of the Gelfand-Mazur theorem for $F^{*}$-algebras in the sense that for any maximal ideal $\mathrm{m}$ in $A$ the algebra $A / \mathrm{m}$ will be isomorphic to the algebra of complex numbers.

Proof. The algebra $A^{R}$ is an archimedean lattice-ordered real algebra with order unit $e$ (= the function equal to 1 everywhere) which is complete in the norm introduced by Kadison [2, p. 5], since this norm reduces to the uniform norm in the case of a function algebra. We are therefore in position to apply Kadison's full representation theorem for ordered algebras (Theorem 3.1 in [2]. This theorem is originally due to Stone). Thus $A^{R}$ is isomorphic to $C^{R}(\mathfrak{B})$ under an isomorphism which preserves the algebra, order and hence the norm structure of $A^{R}$. Since an $F^{*}$-algebra is closed with respect to taking complex conjugates it follows that

$$
f=f_{1}+i f_{2} \in A \quad \text { if and only if } f_{1}, f_{2} \in A^{R} .
$$

Due to this the given isomorphism $A^{R} \cong C^{R}(\mathfrak{P})$ may be extended by linearity to a complex algebra isomorphism $A \cong C(\mathfrak{P})$. That this extended 
ismorphism is norm-preserving can be seen as follows: Any point $a \in \mathbf{X}$ defines a unique $\mathfrak{m}_{a} \in \mathfrak{P}$ by setting

$$
\mathfrak{m}_{a}=\left\{f ; f \in A^{R} \& f(a)=0\right\} .
$$

Denoting this mapping $a \rightarrow \mathfrak{m}_{a}$ of $X$ into $\mathfrak{B}$ by $\varphi$ we see that $\varphi(X)$ is dense in $\mathfrak{P}$. For otherwise there would exist a non-zero function in $C^{R}(\mathfrak{B})$ corresponding to the zero-function in $A^{R}$ by the given isomorphism $f \rightarrow \hat{f}$ from $A^{R}$ onto $C^{R}(\mathfrak{B})$ and this is impossible. Moreover all the functions $\hat{f}$ are continuous and $f(a)=\hat{f}\left(\mathrm{~m}_{a}\right)$ according to the convention made above. The preservation of the norm now easily follows from these two facts together with the density of $\varphi(X)$ in $\mathfrak{B}$. From this norm-preserving algebra isomorphism $A \cong C(\mathfrak{P})$ we get a one-to-one mapping between the corresponding families of maximal ideals $\mathfrak{M}$ and $\mathfrak{M}^{\prime}$. It is further, since $\mathfrak{P}$ is compact, an elementary fact that there is one-to-one correspondence between $\mathfrak{B}$ and $\mathfrak{M}^{\prime}$ and that this correspondence is a homeomorphism when both spaces are equipped with the Gelfand topology. We may therefore first replace $\mathfrak{P}$ by $\mathfrak{M}^{\prime}$ and afterwards $\mathfrak{M}^{\prime}$ by $\mathfrak{M}$ so as to arrive at the desired conclusion $A \cong C(\mathfrak{M})$.

We note that the above proof does not use the fact that an $F^{*}$-algebra is closed under the operation of taking absolute values. This will, however, be used in our second proof.

3. The second proof of the theorem. We shall now offer a second and entirely elementary proof of the Gelfand-Neumark theorem for $F^{*}$-algebras. This proof relies only on the bare rudiments of Kadison's theory. In fact we need only an analogue of the representation theorem announced in [2] as Lemma 2.5. (Even in the self-contained exposition of Kadison containing all definitions and preparations the proof of this lemma is completed within three pages.) This result which is stated below as Lemma 1 is not a full representation theorem and thus less favourable as a starting point for a proof than the full representation theorem used in our first proof. In fact the lack of a full representation (i.e. by all the functions in $C^{R}(\mathfrak{N})$ ) causes some difficulty in proving that $\varphi(X)$ is dense in $\mathfrak{N}$ in the Gelfand topology. And this fact is even more essential to us here as we start only with an ordered vector space representation and have to introduce the multiplication in $A$ by an extension by continuity. But this difficulty can be overcome by using the fact that the definition of the Gelfand topology in $\mathfrak{N}$ in case of $F^{*}$-algebras can be given a different formulation where the density of $\varphi(X)$ in $\mathfrak{N}$ can be easily seen. We proceed to fill in the details and start by announcing the relevant representation theorem 
Lemma 1. Any archimedean lattice-ordered vector space $E$ over the reals with an order unit can be represented isomorphically, isometrically, and with preservation of order as a vector space of real-valued continuous functions on the compact space of all maximal convex lattice-closed subspaces of $E$ equipped with the Gelfand topology.

For definitions and proof we refer the reader to [2]. We remark that the above lemma is slightly different from Kadison's Lemma 2.5 inasmuch as he uses general (not necessarily lattice-closed) convex subspaces. It is, however, easy to verify that his proof goes through also with our additional restriction on the convex subspaces. Let us only indicate briefly the necessary changes in Kadison's proof. Instead of his Lemma 2.2 we may refer the reader to $[1$, p. 239] where it is remarked that $R$ is the only real lattice-ordered vector space without proper convex lattice-closed subspaces (1-ideals in the terminology of [1]). Lemma 2.3 where Kadison's norm is introduced carries over without change. As to Lemma 2.4 we only need to verify that the set $I_{\alpha}$ is also lattice-closedand this is immediate. In fact if $\gamma_{1}$ and $\gamma_{2}$ can be used for $b_{1}$ and $b_{2}$ respectively then $\gamma_{1}+\gamma_{2}$ can be used for $b_{1} \cup b_{2}$. That finally $\mathfrak{B}$ (now meaning the set of all positive normed linear functionals preserving union) is $w^{*}$-closed (i.e. closed in the Gelfand topology) is obvious.

We now introduce a closure operation into the famly $\mathfrak{R}$ of all maximal convex lattice-closed subspaces of the lattice-ordered vector space $A^{R}$ of all real-valued functions in the $F^{*}$-algebra $A$. If $\mathfrak{A} \subseteq \mathfrak{N}$ we define the closure $\overline{\mathfrak{A}}$ of $\mathfrak{A}$ by

$$
\overline{\mathfrak{A}}=\left\{\mathfrak{n} ; \mathfrak{n} \subseteq \overline{\bigcup_{\mathfrak{n} \in \mathfrak{A}} \mathfrak{n}_{i}}\right\}
$$

where the bar on the right denotes closure in $A^{R}$ with respect to the uniform norm topology. The key lemma in order to establish the essential properties of the operation $\mathfrak{A} \rightarrow \overline{\mathfrak{A}}$, in particular to prove that it defines a topology, is the following

Lemma 2. A necessary and sufficient condition that $\mathfrak{n}_{0} \in \overline{\mathfrak{A}}$ is that

for all $f \in \mathfrak{n}_{\mathbf{0}}$.

$$
\inf _{\mathfrak{n} \in \mathfrak{A}}|\hat{f}(\mathfrak{n})|=0
$$

Proof. Suppose that $\mathfrak{n}_{0} \in \overline{\mathfrak{A}}$, that is, $\mathfrak{n}_{0} \subseteq \overline{\bigcup_{\mathfrak{n}_{i} \in \mathfrak{U}} \mathfrak{n}_{i}}$. This means that to any $f \in \mathfrak{n}_{0}$ we can find a function $g$ belonging to a suitable $\mathfrak{n}_{i_{0}} \in \mathfrak{A}$ such that

$$
\|f-g\|_{\infty}<\varepsilon
$$

for any $\varepsilon>0$ given in advance. By the isometry of Lemma 1 this gives 


$$
\|\hat{f}-\hat{g}\|_{\infty}<\varepsilon
$$

which implies $\left|\hat{f}\left(\mathfrak{n}_{i_{0}}\right)\right|<\varepsilon$ because of $\hat{g}\left(\mathfrak{n}_{i_{0}}\right)=0$. Thus

Suppose conversely that

$$
\inf _{\mathfrak{n} \in \mathfrak{U}}|\hat{f}(\mathfrak{n})|=0 \text {. }
$$

$$
\inf _{\mathfrak{n} \in \mathfrak{A}}|\hat{f}(\mathfrak{n})|=0 \quad \text { for all } \quad f \in \mathfrak{n}_{\mathbf{0}} .
$$

Given $f \in \mathfrak{n}_{0}$ this implies the existence of a sequence $\left\{\mathfrak{n}_{n}\right\} \subseteq \mathfrak{A}$ such that

Therefore

$$
\hat{f}\left(\mathfrak{n}_{n}\right)=\alpha_{n} \rightarrow 0 \quad \text { as } \quad n \rightarrow \infty .
$$

$$
f=\lim _{n \rightarrow \infty}\left(f-\alpha_{n} e\right)
$$

where $e$ denotes the identity element of $A$ ( $e$ is also the order unit of $A^{R}$ ). Since

this implies that

$$
\left(\widehat{f-\alpha_{n} e}\right)\left(\mathfrak{n}_{n}\right)=0 \quad \text { or } \quad f-\alpha_{n} e \in \mathfrak{n}_{n}
$$

$$
f-\alpha_{n} e \in \bigcup_{\mathfrak{n}_{i} \in \mathfrak{A}} \mathfrak{n}_{i} \quad \text { for all } n .
$$

(1) and (2) now give the desired conclusion.

Lemma 3. The operation $\mathfrak{A} \rightarrow \overline{\mathfrak{A}}$ defines a topology in $\mathfrak{N}$ such that all the functions $\hat{f} \in \widehat{A^{R}}$ are continuous in this topology.

Proof. The verification of $\mathfrak{A} \subseteq \overline{\mathfrak{A}}, \overline{\overline{\mathfrak{A}}}=\overline{\mathfrak{A}}, \mathfrak{A} \subseteq \mathfrak{B} \Rightarrow \overline{\mathfrak{A}} \subseteq \overline{\mathfrak{B}}$ and $\overline{\mathfrak{A}} \cup \overline{\mathfrak{B}}$ $\subseteq \overline{\mathfrak{A} \cup \mathscr{\mathfrak { B }}}$ is immediate. Assume therefore that $\mathfrak{n}_{\mathbf{0}} \notin \overline{\mathfrak{A}} \cup \overline{\mathfrak{B}}$, thus

$$
\inf _{\mathfrak{n} \in \mathfrak{A}}|\hat{f}(\mathfrak{n})|>0 \quad \text { and } \quad \inf _{\mathfrak{n} \in \mathfrak{A}}|\hat{g}(\mathfrak{n})|>0
$$

for suitable $f, g \in \mathfrak{n}_{0}$. Since $\mathfrak{n}_{0}$ is lattice-closed $f \in \mathfrak{n}_{0}$ implies $|f| \in \mathfrak{n}_{0}$. Therefore

proving that

$$
\inf _{\mathfrak{n} \in \mathfrak{A} \cup \mathfrak{B}}(\mid \widehat{f|+| g \mid})(\mathfrak{n})>0 \quad \text { with } \quad|f|+|g| \in \mathfrak{n}_{0}
$$

$$
\mathfrak{n}_{\mathbf{0}} \notin \overline{\mathfrak{A} \cup \mathfrak{B}} \text {. }
$$

In order to prove that $\hat{f}$ is continuous on $\mathfrak{N}$ in the above topologywhich we shall call the $F^{*}$-topology-we first remark that any set of the form

$$
\mathfrak{D}=\{\mathfrak{n} ;|\hat{f}(\mathfrak{n})|<\alpha\}
$$

is open in the $F^{*}$-topology for any fixed $f \in A$ and $\alpha>0$. In fact if $\mathfrak{n}_{0} \in \mathfrak{D}$ we have $\hat{f}\left(\mathfrak{n}_{0}\right)=\beta$ with $|\beta|<\alpha$. Now 


$$
f-\beta e \in \mathfrak{n}_{0} \quad \text { and } \inf _{\mathfrak{n} \in \mathfrak{R}-\mathcal{D}}|(\widehat{f-\beta e})(\mathfrak{n})|>0
$$

proving that $\mathfrak{n}_{0} \notin \mathfrak{N}-\mathfrak{D}$ and $\mathfrak{D}$ is open. The continuity of $\hat{f}$ at $\mathfrak{n}_{0}$ then follows immediately from the fact that the set

$$
\left\{\mathfrak{n} ;\left|\left(\widehat{\hat{f}\left(\mathfrak{n}_{0}\right) \cdot e-f}\right)(\mathfrak{n})\right|<\varepsilon\right\}=\left\{\mathfrak{n} ;\left|\hat{f}\left(\mathfrak{n}_{0}\right)-\hat{f}(\mathfrak{n})\right|<\varepsilon\right\}
$$

is open in the $F^{*}$-topology.

Lemma 4. The $F^{*}$-topology in $\mathfrak{R}$ coincides with the Gelfand topology.

Proof. Since the Gelfand topology in $\Re$ is characterized as the coarsest topology which makes all the functions $\hat{f}$ with $f \in A^{R}$ continuous it follows from Lemma 3 that the $F^{*}$-topology is finer than the Gelfand topology. Let therefore $\mathfrak{A}$ be a closed subset of $\mathfrak{R}$ in the $F^{*}$-topology, i.e.

$$
\mathfrak{A}=\left\{\mathfrak{n} ; \mathfrak{n} \subseteq \overline{\bigcup_{\mathfrak{n}_{i} \in \mathfrak{M}} \mathfrak{n}_{i}}\right\}
$$

To any $\mathfrak{n}_{0} \notin \mathfrak{A}$ we can therefore find an $f \in \mathfrak{n}_{0}$ such that

This gives

$$
f \notin \bigcup_{\mathfrak{n}_{i} \in \mathfrak{M}} \mathfrak{n}_{i}
$$

$$
\inf _{\mathfrak{n} \in \mathfrak{Q}}|\hat{f}(\mathfrak{n})|=\alpha>0 \quad \text { and } \quad \hat{f}\left(\mathfrak{n}_{0}\right)=0 .
$$

The function $|\hat{f}|$ being continuous in the Gelfand topology the set $\{\mathfrak{n} ;|\hat{f}|(\mathfrak{n}) \geqq \alpha\}$ will be a closed set in the Gelfand topology which contains $\mathfrak{A}$ and excludes $\mathfrak{n}_{0}$. This shows that $\mathfrak{A}$ as an intersection of closed sets is also closed in the Gelfand topology.

Lemma 5. The set $\varphi(X)$ is dense in $\mathfrak{R}$ in the Gelfand topology.

Proof. According to Lemma 4 it suffices to show the density of

$$
\varphi(X)=\left\{\mathfrak{n}_{a} ; \mathfrak{n}_{a}=\left\{f ; f \in A^{R} \& f(a)=0\right\}\right\}
$$

in $\mathfrak{N}$ in the $F^{*}$-topology, i.e. that

$$
\overline{\varphi(X)}=\left\{\mathfrak{n} ; \quad \mathfrak{n} \subseteq \overline{\bigcup_{\mathfrak{n}_{a} \in \varphi(X)} \mathfrak{n}_{a}}\right\}
$$

is all of $\mathfrak{R}$. Now, the right hand side of (3) consists exactly of those $f \in A^{R}$ such that

So if

$$
\inf _{x \in X}|f(x)|=0 .
$$

$$
\mathfrak{n}_{\mathbf{0}} \neq \overline{\bigcup_{\mathfrak{n}_{a} \in \varphi(X)} \mathfrak{n}_{a}}
$$


this means that $\mathfrak{n}_{0}$ contains an $f_{0}$ with

$$
\inf _{x \in X}\left|f_{0}(x)\right|=\alpha>0 .
$$

Then also $n f_{0} \in \mathfrak{n}_{0}$ for any integer $n>0$ and $\mathfrak{n}_{0}=A^{R}$ by the convexity of $\mathfrak{n}_{0}$ and the boundedness of the functions in $A^{R}$. This proves the lemma.

With these preparations we can easily finish the second proof of the theorem. By Lemma 1 we have a one-to-one mapping of the ordered vector space $A^{R}$ onto $\widehat{A^{R}} \subseteq C^{R}(\Re)$ which preserves all structure. The inverse mapping of $\widehat{A^{R}}$ onto $A^{R}$ is essentially the restriction of the functions to a dense subset. This makes it possible to extend the isomorphism $A^{R} \rightarrow \widehat{A^{R}}$ to an algebra-isomorphism in an obvious way. Since $A^{R}$ is complete and the mapping is norm-preserving it follows that $\widehat{A^{R}}$ is a complete algebra of real-valued continuous functions on the compact Hausdorff space $\mathfrak{R}$ which separates points. According to the StoneWeierstrass theorem we therefore have the algebra-isomorphism

$$
A^{R} \cong C^{R}(\mathfrak{M})
$$

and we can now argue with $\mathfrak{R}$ just as we did with $\mathfrak{P}$ in the first proof in order to complete our second proof.

REMarks. It is possible that the topological part of our second proof can be simplified further. One could either try to reason directly with the Gelfand topology in its original form or to use the Stone topology (called the hull-kernel topology in [3]). In the Stone topology the density of $\varphi(X)$ in $\mathfrak{A}$ is obvious and as the Stone topology in $\mathfrak{N}$ is known to be coarser than the Gelfand topology it would be sufficient to establish that the Stone topology in $\mathfrak{N}$ is Hausdorff. Since $\mathfrak{R}$ is a $T_{1}$-space in the Stone topology it would therefore also be sufficient to verify that it is possible to introduce a group-operation into $\mathfrak{N}$ such that $\mathfrak{R}$ is a topological group with respect to the Stone topology.

4. Added in proof. We have recently observed that there already exists a quite simple proof of the Gelfand-Neumark theorem in the case of function algebras. (See: Dunford and Schwartz: Linear operators, pp. 274-75.) But this proof is different from the two proofs given above, besides that the $F^{*}$-topology for ordered vector spaces may have an independent interest. This topology has already been used occasionally in the case of Banach algebras. 


\section{REFERENCES}

1. G. Birkhoff, Lattice theory, New York, 1948.

2. R. V. Kadison, A representation theory for commutative topological algebra, Mem. Amer. Math. Soc. No. 7 (1951).

3. L. H. Loomis, An introduction to abstract harmonic analysis, New York, 1953.

4. A. Weil, Sur les fonctions presque périodiques de von Neumann, C. R. Acad. Sci. Paris 200 (1935), 38-40.

THE INSTITUTE FOR ADVANCED STUdY, PRINCETON, N.J., U.S.A. $\triangle N D$

UNIVERSITY OF OSLO, NORWAY 